\title{
Diacronie
}

Studi di Storia Contemporanea

$N^{\circ} 7,3 \mid 2011$

«Spagna Anno Zero»: la guerra come soluzione

\section{Fenomenología y Guerra Civil española}

La visibilidad de las fuentes documentales sobre las Brigadas

Internacionales (1937-2011)

Lourdes Prades-Artigas y Montserrat Sebastiá-Salat

\section{(2) OpenEdition}

Journals

Edición electrónica

URL: http://journals.openedition.org/diacronie/3287

DOI: 10.4000/diacronie.3287

ISSN: 2038-0925

Editor

Association culturelle Diacronie

Referencia electrónica

Lourdes Prades-Artigas y Montserrat Sebastiá-Salat, « Fenomenología y Guerra Civil española », Diacronie [En línea], № 7, 3 | 2011, documento 12, Puesto en línea el 29 julio 2011, consultado el 03 mayo 2019. URL : http://journals.openedition.org/diacronie/3287 ; DOI : 10.4000/diacronie.3287 


\section{Diacronie}

N. 7 | 7|2011 Spagna Anno Zero: la guerra come soluzione

12/

\section{Fenomenología y Guerra Civil española: La visibilidad de las fuentes documentales sobre las Brigadas Internacionales (1937-2011)}

Lourdes PRADES-ARTIGAS*

Montserrat SEBASTIÁ-SALAT**

Artículo que analiza y tipifica la producción histórica, literaria, política, sociológica, periodística, cinematográfica y museística sobre las Brigadas Internacionales y los Brigadistas en el contexto actual a favor de la recuperación de la memoria histórica sobre la Guerra Civil española. Tratamos las fuentes históricas con el mismo rigor científico y objetivo que las de las ciencias experimentales, y llegamos a diversas conclusiones sobre la complejidad de dichas fuentes como son que el contenido de las fuentes históricas no presenta ninguna dificultad real para explorar su coherencia interna y sus interrelaciones, a pesar de la subjetividad que las caracteriza; $y$ asimismo, proponemos otra metodología de análisis que se distancia de las técnicas y de los sistemas de análisis de otras áreas del conocimiento, como son la psicología o la estadística matemática, y construimos una metodología de investigación propia para las fuentes históricas. Nuestro objetivo es la accesibilidad de las fuentes en los sistemas digitales y en la Web para garantizar la investigación, la docencia y la recuperación de la memoria colectiva sobre la Guerra Civil española y sus protagonistas. 


\section{Introducción}

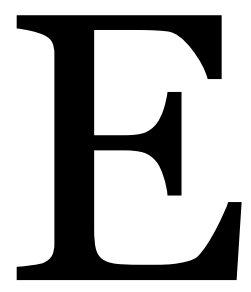

ntre 1936 y 1939, y también durante los años posteriores, la Guerra Civil española se convirtió en un símbolo de la lucha contra el fascismo y también en la esperanza de llevar a cabo una verdadera revolución social. La contienda fue considerada por muchos como la última gran causa, el último conflicto en el que se luchó por unos ideales, perfectamente escenificados por la presencia en España de miles de brigadistas llegados voluntariamente de todo el mundo. En definitiva, era la primera confrontación a escala internacional entre los fascismos y las democracias, el ensayo general de la que sería poco después la Segunda Guerra Mundial.

Entre todas las víctimas de la Guerra civil española están las fuentes documentales. Con la victoria del fascismo y la consolidación de la dictadura franquista durante 40 años, al nuevo régimen no le interesa la accesibilidad de las fuentes, se asegura que en la legislación sobre la consulta de los archivos y las bibliotecas esté implícita la peligrosidad de la historiografía, y así las fuentes documentales sufren la lógica del silencio y la dispersión. Y por encima de otros temas en la historiografía de la guerra española hay uno de maldito que es el fenómeno de los brigadistas y las Brigadas Internacionales, porque en su historia subyace la identificación entre el mito y la leyenda, entre el comunismo y el intervencionismo extranjero. Por todo ello, y a pesar de los 75 años transcurridos, son pocas las fuentes documentales que afrontan este fenómeno histórico en su totalidad y con el rigor que precisa la historiografía de la memoria colectiva sobre la identidad de más de 35.00o personas y su impacto en la Guerra Civil española.

El análisis fenomenológico de las fuentes documentales sobre la Guerra Civil española y sobretodo acerca de las Brigadas Internacionales y los brigadistas es muy complejo. Dado que debe afrontar la heterogeneidad, la diversidad de la tipología, las ideologías, el uso propagandístico, la irregularidad en su publicación, la evolución política en España, y la evolución de los bloques políticos creados después de la II Guerra Mundial. Por otra parte, estas fuentes documentales ponen en evidencia los problemas de acceso a las fuentes primarias por parte de los historiadores; también su dispersión en asociaciones, archivos públicos y patrimoniales, bibliotecas, centros de investigación, y unidades de información. Y por último, sorprende la invisibilidad de las fuentes en los sistemas digitales y que sólo se corrige actualmente con la irrupción de las políticas a favor de la recuperación de la memoria. 
En este estudio el objetivo es la visibilidad de las fuentes documentales sobre las Brigadas Internacionales y los Brigadistas de la Guerra Civil española a partir de dos proyectos: el diseño de un prototipo de sistema digital sobre las Brigadas Internacionales y los Brigadistas creado por Lourdes Prades en su tesis doctoral, y el proyecto de creación del primer Portal semántico sobre las Brigadas Internacionales y los Brigadistas dirigido por Montserrat Sebastià y en que también participa Lourdes Prades ${ }^{1}$.

\section{Interpretaciones historiográficas sobre las Brigadas Internacionales}

En el año 2003 se celebra en Cuenca la mesa redonda sobre las Brigadas Internacionales $^{2}$ y se establece una clasificación muy interesante sobre las líneas historiográficas que podían definirse sobre el tema. Según ésta nomenclatura, podemos dividir los diversos estudios existentes en tres enfoques principales:

1. La línea "franquista", ejemplo de la cual es Ricardo de la Cierva, que se define por su ataca constante al comunismo;

2. La línea "aséptica" o "ecléctica", que si bien reconoce que los brigadistas eran voluntarios, da más trascendencia al control de Moscú sobre éstos hombres;

3. La línea de los "historiadores", que reconoce abiertamente la importancia y la repercusión que tuvieron las Brigadas Internacionales, y quizá son los únicos que ofrecen una contextualización tanto dentro del ámbito geográfico español como también en el internacional.

Las múltiples interpretaciones existentes, tanto sobre la guerra como sobre los voluntarios que lucharon en ella, difieren enormemente en función del período histórico en el cual se escribieron los documentos que lo atestiguan. La producción literaria sobre el fenómeno brigadista entre 1936 y 1939 se basa únicamente en definir cuál era el papel que representan en el conflicto bélico los voluntarios que llegaban a España. Así pues, con respecto al bando republicano, se trata de héroes que defienden las libertades de los españoles mientras que el bando fascista los define como un grupo de bolcheviques que debían ser exterminados para evitar que España cayese en sus

URL: < http://www.sidbrint.org >.

2 REQUENA GALLEGO, Manuel, SEPÚlVEDA LOSA, Rosa Ma. (coords.), Las Brigadas Internacionales: el contexto internacional, los medios de propaganda, literatura y memorias, Cuenca, Ediciones de la Universidad de Castilla-La Mancha, 2003. (La luz de la memoria ; 1). 
manos. No podemos olvidar que durante los años del franquismo no existe ningún tipo de interpretación historiográfica sobre el papel que tienen estos voluntarios en la guerra. Las pocas fuentes que se publican y que tratan el tema, evidentemente lo hacen desde la óptica de los vencedores afirmando que la cruzada católica ha logrado frenar el avance comunista favorecido por la llegada de los internacionales.

A partir de 1975, con la muerte del dictador y el inicio de la transición democrática, se establece un pacto de silencio para evitar que vean la luz verdades que se habían escondido durante 40 años. Se piensa que es el momento de enterrar los odios y antiguas rivalidades y que, por lo tanto, no es adecuado empezar a editar textos que ofrezcan nuevas versiones sobre la interpretación tradicional y franquista de la Guerra Civil española.

No será hasta finales de la década de los 80 cuando empieza a aparecer un nuevo discurso de aquello que acaeció entre 1936 y 1939, más objetivo, más documentado y por lo tanto, más real. También a partir de este momento empiezan a celebrarse actos conmemorativos y de reconocimiento de personas, instituciones y grupos que participaron al lado del bando republicano en los duros momentos de la contienda.

\section{Historiografía sobre les Brigadas Internacionales (1936-2010)}

El número de publicaciones sobre la Guerra Civil española supera actualmente los 50.00o títulos, cifra sólo superada por la que generó la Primera Guerra Mundial3. Ésta necesidad de escribir sobre la guerra española debemos interpretarla en el contexto histórico en el que se produjo: fue la última guerra revolucionaria en Europa y los intelectuales de ambos bandos manifestaron su compromiso por la causa mediante su aportación escrita. Durante los primeros meses del año 1937 se publicó la obra de André Malraux L'Espoir4, en Francia, y No pasaran! : they shall not pass : a story of the Battle of Madrid 5 de Upton Sinclair, en los Estados Unidos. El año siguiente la producción bibliográfica se incrementó y varió el contenido. La guerra avanzaba y el aumento de experiencias y vivencias personales se reflejó en las obras que se publicaban, relatos personales y biografías noveladas. Son un ejemplo Spanish testament $^{6}$ de Arthur Koestler, o la obra de Georges Bernanos Les grands cimentières

3 First World War Studies.

URL: < http://www.firstworldwarstudies.org/ > [visitado el 14/03/2011].

4 MALRAUX, André, L'Espoir, Paris, Gallimard, 1937.

5 SINCLAIR, Upton, No pasaran! they shall not pass!: a story of the Battle of Madrid, Pasadena (Calif.), The Author, 1937.

$6 \quad$ KOESTLER, Arthur, Spanish testament, London, Left Book Club Edition - Gollancz, 1937. 
sous la lune7. Por último, las obras publicadas en el año 1939 son las menos conocidas, exceptuando la de Hemingway For whom the wells tolls ${ }^{8}$ que su fama la llevará hasta la gran pantalla. Posteriormente, la historiografía sobre la Guerra Civil española ha evolucionado y han participado en ella historiadores relevantes tanto españoles como extranjeros.

Un caso diferente es la historiografía específica sobre las Brigadas Internacionales. La producción bibliográfica sobre este tema sigue básicamente cuatro tendencias heterogéneas y, a menudo, antagónicas:

1.Las obras de los protagonistas (los brigadistas)

2. La producción de la organización de las Brigadas Internacionales y la prensa interbrigadista

3. La producción franquista

4. La producción extranjera

Recordemos que la situación europea durante los años 30 estableció una estrecha relación entre la crisis española y la de la Europa de entreguerras. El golpe de estado del 18 de julio de 1936 en España supuso para el resto de países europeos la cristalización de los temores hacia el fascismo. En este contexto se entiende que gran parte de los brigadistas que vinieron no lo hicieran como consecuencia de una propaganda organizada, sino siguiendo su propio impulso. Los italianos exiliados en Francia se enfrentaban así a Mussolini, los alemanes perseguidos por los nazis se levantaban en contra del tercer Reich; los anglosajones y los franceses iban en contra de los alemanes; los checoslovacos luchaban por Praga. Fue a posteriori, que ante esta situación, la Komintern, con el apoyo de Stalin, decidió crear las Brigadas Internacionales en septiembre de 1936. Esto supuso la creación de un ejército internacional, único en la historia de cualquier país, tanto en lo referente al número de efectivos como por el hecho de venir a España de forma voluntaria. Los estudios más sólidos afirman que fueron más de 35.00o los brigadistas que lucharon al lado del ejército republicano y que procedían de más de 50 países distintos 9.

7 BERNANOS, Georges, Les grands cimetières sous la lune, Paris, Plon, 1938.

8 HEMINGWAY, Ernest, For whom the bell tolls, New York, Charles Scribner's Sons, 1940.

9 Aquí un breve listado de las primeras obras dedicadas a las Brigadas Internacionales, según el país de procedencia de la mayoría de los voluntarios. Polonia: AJZNER, Seweryn, Polska a wojna domowa $w$ Hiszpanii 1936-1939, Warszawa, Pa'nstwowe Wydawnictwo Naukowe, 1968; Francia: GILLAIN, Nick, Le Mercenaire, Paris, Fayard, 1938; Brasil: CUNHA, José Gay, Um Brasileiro na guerra española, Rio de Janeiro, Livraria do Globo, 1946, 4 (Documentos de nossa época, $\mathrm{n}^{\mathrm{o}} 33$ ); Gran Bretaña: GURNEY, Jason, Crusade in Spain, London, Faber and Faber, 1974; Estados Unidos: REGLER, Gustav, The great crusade, New York, Longmans Green 
Este fenómeno, tan extraordinario por los motivos ya señalados, generó una importante producción bibliográfica, que se tradujo en un gran número de novelas, memorias y estudios históricos que tenían en común una clara preferencia por uno u otro bando. El espíritu que impregna estos textos es profundamente intimista. Los relatos interbrigadistas nunca se caracterizaron por su triunfalismo: las victorias siempre estaban impregnadas de un cierto pesimismo y las derrotas eran numerosas y continuadas. Por ello eran conscientes que estaban luchando en una guerra que estaban perdiendo irreversiblemente. Entre 1936 y 1939, del mismo modo que se escribió sobre cualquier aspecto de la guerra, también se editó un gran número de publicaciones sobre las Brigadas Internacionales. Mucha de esta bibliografía es claramente propagandista e impulsada por el Comité de Propaganda y por el Comisionado de las Brigadas ${ }^{10}$, como es el caso de Un año en las Brigadas Internacionales ${ }^{11}$.

El primer estudio que analiza la producción bibliográfica generada sobre las Brigadas Internacionales es la obra de Lizón Gadea ${ }^{12}$. El aspecto más destacable es que se trata de una obra pionera en tratar el tema, sin embargo tiene muchas limitaciones historiográficas. Más próximos a nuestros días, podemos destacar los estudios de Michael Alpert ${ }^{13}$ y Enrique Moradiellos ${ }^{14}$, aunque el mejor trabajo sobre este tema es el de Manuel Requena ${ }^{15}$. Es un estudio extraordinariamente bien estructurado donde se hace un repaso de las obras que el tema brigadista suscitó, a la vez que reflexiona sobre

and Co., 1940; Cuba: TORRIENTE BRAU, Pablo, Peleando con los milicianos, La Habana, Nuevo Mundo, 1962; Argentina: OSSELLI, Carlo, Acción y carácter: escritos políticos y autobiográficos, Buenos Aires, Americalee, 1944; Holanda: LAST, Jef, Brieven uit Spanje, Amsterdam, Contact, 1937[?]; Alemania: MAASSEN, Hans, Brigada Internacional ist unser Ehrenname. Erlebnisse ehemaliger deutscher Spanienkämpfer, 2 vols., Berlin, Militärverlag der Deutschen Demokratischen Republik, 1974; Italia: LONGO, Luigi, Le Brigate Internazionali in Spagna, Roma, Riuniti, 1956; Checoslovaquia: PRIBYL, Zdenek, La corrida. Príbeny ceského malíre ve spanelske válce, Praha, Nase vojsko, 1960; México: DE VRIES, Lini M., Espagne 1937, memorias, Xalapa, Universidad Veracruzana, 1965; Rumanía: ROMAN, Valter, Evocâri, Bucuresti, Editura Eminescu, 1980; Suecia: WEICKERT, Eugen, Frihetens soldater: minnen fran en demokratis kamp mot den europeiska fascismen 1936-1939, Malmö, Acupress, 1974.

10 BALK, Theodor, La Quatorzième : d'après des rapports, des conversations, des carnets de notes, Madrid, Éditions du Commissariat des Brigades Internationales, 1937; Nos combats contre le fascisme. Le livre de la 15eme Brigade Internationale sur le front d'Espagne, Madrid, [s.n.], 1937, p. 16.

${ }^{11}$ Un año de las Brigadas Internacionales. [S.l.], Edición del Comisariado Político de las Brigadas Internacionales, 1937, p. 108.

12 LIZÓN GADEA, Adolfo, Brigadas Internacionales en España, Madrid, Editora Nacional, 1940.

13 ALPERT, Michael, El ejército republicano en la Guerra Civil, París, Ruedo Ibérico Barcelona, Ibérica de Ediciones y Publicaciones, 1968; ALPERT, Michael, A new international history of the Spanish Civil War, London, Macmillan, 1994.

14 MORADIELLOS, Enrique, El reñidero de Europa: las dimensiones internacionales de la Guerra Civil española, Barcelona, Península, 2001.

15 REQUENA GALLEGO, Manuel, «Las Brigadas Internacionales: una aproximación historiográfica», Ayer, $\mathrm{n}^{\circ}$ 56, 2004, pp. 11-36. 
qué autores han tratado el tema y cuáles eran sus intenciones al hablar sobre este aspecto de la Guerra Civil Española.

Al margen del análisis historiográfico y bibliográfico, fueron muchas las obras que se editaron contemporáneamente a los años de guerra, editadas en diferentes idiomas como la obra d'André Marty, Volontaires d'Espagne: douze mois sublimes ${ }^{16}$ y los numerosos libros que describían la actividad de los distintos batallones, divisiones o brigadas, escritos por los propios protagonistas. Estas obras cumplían una doble función, por un lado, dar a conocer cuál era su misión en el contexto bélico en el que participaban y por otro, hacer saber a sus países de origen el verdadero significado de su presencia en España al lado del ejército republicano. Con esta intención se escribieron obras como Authors take sides on the Spanish war ${ }^{17}$, Spanish testament ${ }^{18}$, Boadilla $^{19}$, Volunteer in Spain ${ }^{20}$ o No pasarán! ${ }^{21}$. Mención aparte merece la obra del escritor anarcosindicalista y activista alemán, Rudolf Rocker²2, caracterizada por su clara posición antisoviética.

Durante estos años, la producción bibliográfica producida por el bando franquista fue escasa, por no decir inexistente. Únicamente destacan algunas obras de carácter generalista sobre la Guerra Civil como la de Francesco Belforte ${ }^{23}$ que dedica un volumen a los voluntarios extranjeros a favor de la República. En definitiva, entre 1936 y 1939, la coincidencia de los hechos con la edición de éstas fuentes convierte en inviable la aparición de obras de análisis y de reflexión, y mucho menos, bien documentadas. Los hechos son excesivamente recientes y los sentimientos demasiado a flor de piel para admitir interpretaciones objetivas de un acontecimiento tan complejo y tan trágico.

En la inmediata posguerra las fuentes localizadas siguen teniendo un carácter fundamentalmente propagandístico. No obstante, es precisamente durante estos años cuando el bando vencedor inicia la publicación de una serie de obras con la clara finalidad de acabar con el mito brigadista y mostrar lo que hubiera supuesto para la "España nacional” el triunfo republicano. Destaca la obra casi enciclopédica dirigida

16 MARTY, André, Volontaires d'Espagne: douze mois sublimes, Paris, Comité Populaire de Propagande, [ca. 1937].

17 ARAGON, Louis, Authors take sides on the Spanish war, London, Left Review, 1937.

18 KOESTLER, Arthur, Spanish testament, London, Left Book Club Edition - Gollancz, 1937.

19 ROMILLY, Esmond, Boadilla: a personal record of the English Group of the Thaelmann Battalion of the International Brigade in Spain, London, Hamish Hamilton, 1937.

20 SOMMERFIELD, John, Volunteer in Spain, New York, A. A. Knopf, 1937.

${ }_{21}$ SINCLAIR, Upton, No pasarán! they shall not pass! A story of the Battle of Madrid, cit.

22 ROCKER, Rudolf, Extranjeros en España, Buenos Aires, Imán, 1938.

23 BELFORTE, Francesco, La Guerra Civile in Spagna, Tomo II: gli interventi stranieri nella Spagna rossa, Milano, Istituto per gli Studi di Politica Internazionale, 1938. 
por Joaquín Arraras, Historia de Cruzada Española ${ }^{24}$ y el capítulo dedicado a las Brigadas Internacionales, y la de Adolfo Gadea, Brigadas Internacionales en España ${ }^{25}$. La dura censura sobre lo que se publicaba y la hegemonía de la derecha española posibilitaron que este tipo de material bibliográfico proliferara dentro del país, si bien en el exterior se editaban obras de ideología muy diferente. Muchos brigadistas que volvieron a su país de origen, y en muchos casos participaron en la Segunda Guerra Mundial o sufrieron las purgas estalinistas, son los autores de estas fuentes que datan de los primeros años de la década de los 40. En la mayoría de los casos se trata de memorias y relatos personales donde se autodefinen como luchadores antifascistas y nos explican cuales fueron sus vivencias en el frente, sus temores y las relaciones que establecieron entre ellos. Citamos las obras de Gustav Regler ${ }^{26}$, Sthepen Spender y la más conocida, For whom the bell tolls ${ }^{27}$ que Ernest Hemingway escribió el año 1940 y que fue adaptada al cine sólo un año más tarde.

$\mathrm{Al}$ final de la década de los 40 y primeros años de los 50 empiezan a publicarse en el extranjero y, en menor número dentro de España, las primeras fuentes que pretenden dar una interpretación más historiográfica sobre la actividad de los voluntarios internacionales en la guerra española, en detrimento de la producción de memorias y de narrativa que había caracterizado el período precedente. Se abandona progresivamente la novela y la poesía, muy numerosas durante los años de la guerra, y éstas son substituidas por una nueva producción literaria sobre el tema brigadista basada en memorias ${ }^{28}$ y compilaciones bibliográficas ${ }^{29}$. Fuera del país, se edita en 1956 la obra de Luigi Longo Le Brigate internazionali in Spagna ${ }^{30}$ o L'Épopée d'Espagne ${ }^{31}$.

24 ARRARÁS IRIBARREN, Joaquín, SÁENZ DE TEJADA, Carlos (dirs.), Historia de la cruzada española, Madrid, Ediciones Españolas, 1939-1944.

25 LIZÓN GADEA, Adolfo, Brigadas Internacionales en España, Madrid, Editora Nacional, 1940.

26 REGLER, Gustav, The great crusade, New York - Toronto, Longmans Green and Co., 1940.

27 HEMINGWAY, Ernest, For whom the bell tolls, New York, Charles Scribner's Sons, 1940.

28 Algunos ejemplos de memorias publicados durante los años Cincuenta son: NELSON, Steve, The volunteers: a personal narrative of the figth against fascism in Spain, New York, Masses \& Mainstream, 1953; TORRIENTE BRAU, Pablo, En España, peleando con los milicianos, México, Grijalbo, 1972; WEINERT, Erich, Camaradas: ein Spanienbuch, Berlin, Volk und Welt, 1956; NORTH, Joseph, For valor in battle, New York, Veterans of the Abraham Lincoln Brigade, 1952; BRON, Michal, Pasaremos, Warszawa, Iskry, 1958, p.

29 Algunos ejemplos de colecciones bibliográficas: Épopée d' Espagne: Brigades Internationales 1936-1939, recueil de récits vécus et de documents historiques, París [?], Amicale des Anciens Volontaires Français en Espagne Républicaine, [1956?]; Freiheitskampft des spanischen Volkes und die internationale Solidarität: Dokumente und Bilder zum nationalrevolutionärem Krieg des spanischen Volkes, 1936-1939 - herausgegeben vom Institut für Marxismus-Leninismus beim Zentralkomitee des Sed [Der], Berlin, Dietz, 1956.

3o LONGO, Luigi, Le Brigate Internazionali in Spagna, Roma, Riuniti, 1956.

${ }^{31}$ Épopée d'Espagne : Brigades Internationales 1936-1939 : recueil de récits vécus et de documents historiques, cit.. 
Sin embargo, no fue hasta la década de los sesenta cuando se produjo un giro definitivo en el enfoque y en el contenido de estas obras, ahora mucho mejor documentadas. Son de estos años las obras de Jacques Delperrie de Bayac32, Cecil Eby33, Verle B. Johnston ${ }^{34}$ o Vicent Brome 35.

En España, la dictadura que se instauró en el país al finalizar la guerra dirigía todos los aspectos de la vida económica, social, religiosa y cultural, lo cual se tradujo en un represivo control sobre la producción intelectual, sujeta a la censura. Muchos de los autores que escribieron sobre las Brigadas dentro del Estado entre 1939 y hasta 1975 eran acólitos o simpatizantes del régimen franquista, de otro modo nunca hubiesen podido publicar sus obras. Entre éstos podemos mencionar al ideólogo del régimen Ricardo de la Cierva con la Bibliografía general sobre la guerra de España ${ }^{36} \mathrm{y}$, más concretamente sobre les Brigadas, con Leyenda y tragedia de las Brigadas Internacionales ${ }^{37}$ que reeditó el año 1997 con el título Brigadas Internacionales 19361996: la verdadera historia: mentira histórica y error de estado ${ }^{38}$. Obra escrita con la clara intención de contrarrestar la idea mítica y propagandística que hasta entonces había caracterizado los escritos sobre las Brigadas Internacionales, la mayoría editados en el extranjero. Sin ningún rigor histórico falsifica los números según sus propios intereses y cuantifica el número de brigadistas en 10.000 cuando estudios contemporáneos y actuales hablan de entre 35.000 y 59.00o brigadistas. La edición de 1997 no aporta nada nuevo, es una copia exacta del libro que escribió en 1974 añadiendo un prólogo y dos capítulos en los cuales critica duramente la sesión del Congreso de los Diputados de 1995 cuando se produjo el reconocimiento institucional a las Brigadas Internacionales.

También destacable, Jesús Ma Salas Larrazabal, autor que trata de las acciones bélicas de los aviadores alemanes e italianos en territorio español y se convierte en el principal historiador franquista constructor de los mitos bélicos de postguerra a través

32 DELPERRIE DE BAYAC, Jacques, Les Brigades Internationales, París, Fayard, 1968.

33 EBY, Cecil, Voluntarios norteamericanos en la Guerra Civil española, Barcelona, Acervo, 1974.

34 JOHNSTON B., Verle, Legions of Babel: the International Brigades in the Spanish civil war. University Park (Pa.), Pennsylvania State University Press, 1967.

35 BROME, Vincent, The International Brigades : Spain 1936-1939, London, Heinemann, 1965.

36 DE LA CIERVA, Ricardo (dir.), Bibliografía general sobre la guerra de España, 1936-1939 y sus antecedentes históricos: fuentes para la historia contemporánea de España, vol. XXXIX, Madrid, Secretaría General Técnica del Ministerio de Información y Turismo, 1968.

37 DE LA CIERVA, Ricardo, Leyenda y tragedia de las Brigadas Internacionales: una aproximación histórica a la Guerra Civil española desde las avanzadas del ejército popular, Madrid, Prensa Española, 1974.

38 DE LA CIERVA, Ricardo, Brigadas Internacionales 1936-1996, la verdadera historia: mentira histórica y error de estado, Madridejos, Fénix, 1997. 
de estudios como La guerra de España desde el aire39, la Intervención extranjera en la guerra de España ${ }^{40}$, y la Historia del ejército popular de la República41. Y por último, Enrique Barco Teruel voluntario de la División Azul que, inspirándose en una canción de los voluntarios norteamericanos de la Brigada Abraham Lincoln, publica el libro Valle del Jarama42.

Existen otros autores españoles que, a pesar de no ser historiadores declarados del régimen, consiguieron desarrollar su producción bibliográfica sobre las Brigadas, en pleno Franquismo. Entres estos podemos destacar: José Luis Alcofar Nassaes con Los asesores soviéticos (los mejicanos) en la Guerra Civil española43 y "Spansky": los extranjeros que lucharon en la Guerra Civil44. Ambas publicadas en los años 70 pero, a pesar de ello, condicionadas por el contexto histórico y político de la España de aquellos años. También podemos señalar al historiador Luís Aguilera Duran que, para escribir su libro sobre las Brigadas Internacionales45, viajó a Hungría, Rumanía y Bulgaria para entrevistar a numerosos excombatientes brigadistas y poder consultar documentación en los países de origen sobre el sistema de reclutamiento y organización.

Sin embargo, la obra más representativa de estos años es sin duda la de Andreu Castells Las Brigadas Internacionales de la guerra de España ${ }^{46}$, publicada por primera vez en 1974, cuando ya se veía posible el final de la dictadura franquista Impresor, archivero e historiador, Castells participó en la Guerra Civil en las filas de las Brigadas Internacionales, circunstancia que lo marcó profundamente y lo llevó a escribir la que, en opinión de la mayoría de expertos 47 en el tema, sigue siendo la mejor

39 SALAS LARRAZABAL, Jesús María, La guerra de España desde el aire: dos ejércitos y sus cazas frente a frente, Barcelona, Ariel, 1969.

40 SALAS LARRAZABAL, Jesús María, Intervención extranjera en la guerra de España, Madrid, Editora Nacional, 1974.

41 SALAS LARRAZABAL, Jesús María, Historia del ejército popular de la República, 4 vols, Madrid, Editora Nacional, 1973.

42 BARCO TERUEL, Enrique, Valle del Jarama: Brigada Internacional, Barcelona, Marte, 1969.

43 ALCOFAR NASSAES, José Luis, Los Asesores soviéticos (los mejicanos) en la Guerra Civil española, Barcelona, Dopesa, 1971.

44 ALCOFAR NASSAES, José Luis, "Spansky", los extranjeros que lucharon en la Guerra Civil española, Barcelona, Dopesa, 1973.

45 AGUILERA DURAN, Luís, Orígenes de las Brigadas Internacionales. Madrid, Editora Nacional, 1974, p. 185 (Libros directos ; 20).

46 CASTELLS, Andreu, Las Brigadas Internacionales de la guerra de España, Esplugues de Llobregat, Ariel, 1974, p. 685.

47 Citamos dos ejemplos, el de Raymond Carr que califica este libro como "el último y el mejor estudio sobre las Brigadas" (CARR, Raymond, La tragedia española, la guerra civil en perspectiva, Madrid, Alianza, 1986) y el del escritor franquista Ricardo de la Cierva que lo define como «[el] estudio de Castells, tan riguroso» (DE LA CIERVA, Ricardo, La leyenda de las Brigadas Internacionales, Madrid, Diario El Alcázar, 1969). 
obra general existente sobre las Brigadas Internacionales, referente indispensable para cualquier estudio, y no superada por ninguna otra, más de treinta años después de su publicación. Está ampliamente documentada y contiene un muy completo índice onomástico y una buena bibliografía. Castells la empezó a escribir en 1938, contemporáneamente al conflicto bélico y a las primeras purgas estalinistas de Moscú que afectaron directamente a muchos de los combatientes extranjeros que habían venido a España para luchar. Se trata del primer libro que pretende dar una visión panorámica a este fenómeno y no una visión parcial en función del país de edición de la publicación o de la ideología política de su autor. En definitiva, a pesar de sus limitaciones, marcadas por la falta de medios técnicos en los años en que se escribió, es la obra enciclopédica básica sobre las Brigadas Internacionales.

Exceptuando la obra de Castells, no será hasta finales de la década de los 70 y mucho más durante los años 80, cuando se inicie un nuevo discurso interpretativo de lo que sucedió entre 1936 y 1939, mucho más objetivo y mejor documentado, que se traducirá en la aparición de las primeras obras que podríamos calificar de científicas por la exhaustiva aportación de datos por parte de sus autores, que han visitado archivos, vaciado sistemáticamente fuentes hemerográficas y han hecho revisionismo histórico y metodológico del que todo lo que se había publicado hasta el momento. A partir de estos años empiezan a celebrarse actos conmemorativos y de reconocimiento de diversas personas, instituciones y grupos que participaron al lado del bando republicano durante los duros momentos de la contienda. Coincidiendo con estos actos de reconocimiento, se publicó La llamada española: homenaje a las Brigadas internacionales, 1936-193948, Memorials of the Spanish Civil War49 y el libro sobre la Brigada Lincoln Otra cara de América: los brigadistas y su legado de esperanza ${ }^{50}$. En la actualidad, el tema de las Brigadas Internacionales vuelve a ser objeto de debate y estudio por parte de muchas de las publicaciones que continuamente van apareciendo sobre la Guerra Civil. Han transcurrido 75 años desde el inicio del conflicto, y ahora asistimos al fin de los pocos supervivientes que todavía quedan y la evolución y el estudio de la historia obliga a una reinterpretación de los episodios bélicos, dándoles una lectura mucho más objetiva y metodológicamente más rigurosa.

48 ESTEBAN José, GÓMEZ-PORRO Francisco, REQUENA Manuel (compiladores), La llamada española, homenaje a las Brigadas Internacionales, 1936-1996, Toledo, Ediciones de las Cortes de Castilla-La Mancha, 1996.

49 WILLIAMS Colin, ALEXANDER Bill, GORMAN John, Memorials of the Spanish Civil War, the official publication of the International Brigade Association, Gloucestershire, Alan Sutton, 1996.

50 MORENO José (fotos), GEIST L. Anthony (texto), Otra cara de América : los brigadistas y su legado de esperanza = Passing the torch: the Abraham Lincoln brigade and its legacy of hope, Cádiz, Servicio de Publicaciones - Universidad de Cádiz y Diputación de Cádiz, 2001. 
Los textos que actualmente se editan nada tienen que ver con las primeras publicaciones que hemos analizado en las cuales prevalece el recuerdo y la ideología política del escritor, en detrimento de lo que podríamos denominar "la verdad histórica".

Hoy en día, ninguna publicación que tenga como objeto de estudio las Brigadas Internacionales puede prescindir de autores que, según nuestra opinión, son los grandes especialistas en el tema y que se han dedicado al análisis exhaustivo de algún aspecto concreto del fenómeno brigadista. Entre otros, destacan Mirta Núñez en cuanto a la prensa con sus obras La prensa de guerra en la zona republicana durante la Guerra Civil española, 1936-1939 y las obras que tratan sobre la prensa brigadista51, el estudio de la represión franquista52 y la historia de la mujer53. Fernando Rodríguez de la Torre con la Bibliografía de las Brigadas Internacionales y de la participación de extranjeros a favor de la República (1936-1939) 54, que recopila 2317 referencias bibliográficas. Y sin duda, el historiador Manuel Requena, director del CEDOBI (Centro de Estudios y Documentación de las Brigadas Internacionales) 55 con sus numerosos análisis historiográficos sobre el fenómeno brigadista y su extraordinario monográfico publicado en la revista Ayer ${ }^{6}$ sobre una aproximación bibliográfica de las Brigadas Internacionales.

Otros especialistas actuales que han tratado aspectos concretos de las Brigadas Internacionales son: Magí Crusells y sus estudios sobre la cinematografía, y Maryse Bertrand de Muñoz, experta en literatura de la Guerra Civil, concretamente en la novela. Sus aportaciones más importantes en este campo son las publicaciones $L a$

51 Sobre este tema ha escrito: NÚÑEZ DÍAZ-BALART, Mirta, La prensa de las Brigadas Internacionales, Madrid, [sin ed.], 1983, (Tesis de licenciatura. Universidad Complutense de Madrid, Facultad de Ciencias de la Información, Departamento de Historia, 1983); NÚÑEZ DÍAZ-BALART, Mirta, La disciplina de la conciencia: las Brigadas Internacionales y su artillería de papel, Barcelona, Flor de Viento, 2006.

52 Sobre este tema: NÚÑEZ DÍAZ-BALART, Mirta, "La infancia "redimida" del sistema penitenciaro franquista», en Historia y comunicación social, Facultad de Ciencias de la Información, Departamento de Historia de la Comunicación social (Madrid), $\mathrm{n}^{0} 6$ (2001), pp. 137-148; NÚÑEZ DÍAZ-BALART, Mirta, Consejo de guerra; los fusilamientos en el Madrid de la posguerra, 1939-1945, Madrid, Compañía Literaria, 1997; NÚÑEZ DÍAZ-BALART, Mirta, Los años del terror: la estrategia de dominio y represión del general Franco, Madrid, La Esfera de los Libros, 2004.

53 NÚÑEZ DÍAZ-BALART, Mirta, Mujeres caídas: prostitutas legales y clandestinas en el franquismo, Madrid, Oberón, 2003.

54 RODRÍGUEZ DE LA TORRE, Fernando, Bibliografía de las Brigadas Internacionales y de la participación de extranjeros a favor de la República (1936-1939), Albacete, Instituto de Estudios Albacetenses “Don Juan Manuel” de la Excma. Diputación de Albacete, 2006.

55 CEDOBI:

URL: < http://www.brigadasinternacionales.uclm.es/ > [visitado el 14/03/2011].

56 REQUENA GALLEGO, Manuel, «Las Brigadas Internacionales: una aproximación historiográfica», Ayer, $\mathrm{n}^{\circ}$ 56, 2004, pp. 11-36. 
Guerra Civil española en la novela: bibliografía comentada57 y La novela europea y americana y la Guerra Civil española ${ }^{5}$.

Con el fin de la era soviética y la apertura de los archivos, los investigadores ya disponen de un nuevo campo de estudio para explotar con la incorporación en sus trabajos de información vedada hasta entonces, lo cual posibilita una interpretación más exhaustiva, más real y mejor documentada sobre las Brigadas Internacionales. Ejemplo de éste nuevo enfoque en el estudio del comunismo en general y de los voluntarios internacionales en particular son las obras de Antonio Elorza y Marta Bizcarrondo59, Mary R. Habeck y Grigory Sevostinov ${ }^{60}$, Mrek Jan Chodakiewicz ${ }^{61}$ y Rémi Skoutelsky62, historiador que basó su tesis en los voluntarios franceses de las Brigadas Internacionales ${ }^{6}$. Escribió con Michel Lefebvre, los textos de una extraordinaria recopilación de fotografías de brigadistas ${ }^{64}$, y más recientemente publicó Novedad en el frente ${ }^{65}$.

Un hecho que caracteriza las fuentes escritas durante estos años es que se exploran otros temas de investigación sobre las Brigadas. Se publican libros que hablan sobre la sanidad (La Sanidad en las Brigadas Internacionales) ${ }^{66}$, la vida cotidiana de los brigadistas (La ciudad inventada) ${ }^{67}$, las mujeres (Ve y cuenta lo que pasó en España : mujeres extranjeras en la Guerra Civil : una antología y La brigadista : diario de una dinamitera de la Guerra Civil) ${ }^{68}$, los judíos (iShalom libertad! : judíos en la Guerra

57 BERTRAND DE MUÑOZ, Maryse, La Guerra Civil española en la novela: bibliografía comentada, vol. 3, Madrid, José Porrúa Turanzas, 1982.

58 BERTRAND DE MUÑOZ, Maryse, La novela europea y americana y la Guerra Civil española, Madrid [etc.], Júcar, 1994.

59 ELORZA Antonio y BIZCARRONDO Marta, Queridos camaradas: la internacional Comunista y España, 1936-1939, Barcelona, Planeta, 1999.

6o RADOSH Ronald, R. HABECK Mary y SEVIOSTIANOV Grigory (ed.), España traicionada: Stalin y la Guerra Civil, Barcelona, Planeta, 2002.

61 CHODAKIEWICZ, Marek Jan, Zagrabiona pamie'c. Wojna $w$ Hiszpanii 1936-1939, Warszawa, Froda, 1997, p. 121.

62 SKOUTELSKY, Rémi, L'Espoir guidait leurs pas : les volontaires français dans les Brigades Internationales, 1936-1939, París, Bernard Grasset, 1998.

63 Ibidem.

64 LEFEBVRE Michel, SKOUTELSKY Rémi, Las Brigadas Internacionales: imágenes recuperadas, Barcelona, Lunwerg, 2003.

65 SKOUTELSKY, Rémi, Novedad en el frente: las Brigadas Internacionales en la Guerra Civil, Madrid, Temas de Hoy, 2006.

66 NAVARRO CARBALLO, José Ramón, La Sanidad en las Brigadas Internacionales, Madrid, Servicio de Publicaciones del Estado Mayor del Ejército, 1989.

67 GÓMEZ FLORES, Andrés, La ciudad inventada: Albacete en la Guerra Civil (una historia literaria), Albacete, El Sur, 2002.

68 USANDIZAGA, Aránzazu, Ve y cuenta lo que pasó en España: mujeres extranjeras en la Guerra Civil, una antología, Barcelona, Planeta, 2000; PARSHINA, Elizabeta Aleksandrovna, La brigadista: diario de una dinamitera de la Guerra Civil, Madrid, La Esfera de los Libros, 2002. 
Civil española $)^{69}$, los negros, los latinoamericanos y los homosexuales ${ }^{70}$ o la prensa ${ }^{71}$.

\section{Los autores}

Las fuentes documentales sobre las Brigadas Internacionales son muy heterogéneas, y sus autores pueden ser en muchas ocasiones los mismos protagonistas: los brigadistas.

Podemos clasificar en dos grandes grupos los autores que han escrito sobre las Brigadas Internacionales: los que vivieron la guerra y los que la han interpretado. El legado de los primeros lo constituyen básicamente las memorias y los relatos personales, mientras que el segundo grupo, con más o menos acierto, análisis y rigor histórico, ha producido manuales, tratados e historias parciales, en definitiva, interpretaciones diversas sobre el fenómeno brigadista. Para los escritores extranjeros, tanto aquellos que a la vez eran brigadistas como los investigadores o simpatizantes del tema, el estudio y el análisis de la guerra en general y del tema brigadista en particular, es un hecho lineal en el cual sólo basculan dos sectores, uno el fascismo y el otro la defensa de la libertad. No acostumbran a ir más allá y profundizar en la gran cantidad de implicaciones que éste aspecto de la historia de España tuvo, antes del 18 de julio de 1936 y después, con el inicio de la Segunda Guerra Mundial. Para los autores españoles, el fenómeno de las Brigadas Internacionales no se puede entender al margen de la historia general de la guerra, sus antecedentes y los 40 años de dictadura que le siguieron. A continuación analizamos brevemente las obras de aquellos brigadistas que quisieron dejar por escrito impresiones, temores y deseos de su estancia en España.

\subsection{Ingleses}

Para los escritores ingleses la guerra de España fue una guerra de escritores. El número de ingleses que llegaron a nuestro país para luchar en las Brigadas es

69 LUSTIGER, Arno, iShalom libertad! Judíos en la Guerra Civil española, Barcelona, Flor al Viento, 2001, p. 492 (Del viento terral ; 27).

70 RAMELLA, Pietro, «I 'diversi’ e la guerra di Spagna : ebrei, negri e omosessuali nelle Brigate Internazionali», en L'impegno, Istituto Storico della Resistenza di Borgosesia, $\mathrm{n}^{0} 3$ (2001); GONZÁLEZ, Isidro, Los judíos y la guerra civil española, Madrid, Hebraica Ediciones, 2009, p. 340; URCELAY-MARAGNÈS, Denise, Les volontaires cubains dans la défense de la république espagnole, 1936-1939: la légende rouge, Paris, Harmattan, 2008, p. 288; BAUMANN, Gino, Los voluntarios latinoamericanos en la Guerra Civil española, Cuenca, Ediciones de la Universidad de Castilla-La Mancha, 2009, p. 272 (La luz de la memoria ; 8).

${ }_{71}$ NÚÑEZ DÍAZ-BALART, Mirta, La disciplina de la conciencia: las Brigadas Internacionales y su artillería de papel, Barcelona, Flor de Viento, 2006, p. 287 (70 aniversario Guerra Civil ; 9). 
aproximadamente de unos 2.500 y la producción literaria inglesa relacionada con la guerra es igual o más numerosa. Es en esta extensa producción literaria donde podemos identificar los motivos por los cuales un gran número de europeos y de americanos decidieron tomar posición en el conflicto español. No obstante, muchas de las razones eran específicamente inglesas: el interés de los británicos por lo hispánico y sobre todo por las condiciones sociales y económicas de los años 30. España se convirtió en el destino ideal para los jóvenes de izquierdas comprometidos y dispuestos a luchar contra el fascismo. Estos escritores ingleses, simpatizantes de la causa republicana, se convirtieron en combatientes, periodistas o "turistas accidentales" y muchos de ellos participaron en la actividad política española. En cuanto a su contribución literaria, los más representativos son Auden, Spender, Macneice, Orwell, Slater y Koestler, les obras de los cuáles se han leído hasta las generaciones actuales.

El Partido Comunista, que monopolizaba tanto la propaganda antifascista en Inglaterra como las Brigadas Internacionales en España, prefería ver en sus mítines y en los periódicos a sus escritores e intelectuales más célebres en lugar de conocer de primera mano cual era el día a día en el campo de batalla. Algunos de los autores brigadistas ingleses más destacados son: John Sommerfield autor de Volunteer in Spain $^{72}$; John Cornford escribe unos poemas73; Ralph Fox escritor que escribía para el Daily Worker, órgano del Partido Comunista en Inglaterra; Christopher Caudwell pseudónimo de Christopher St. John Spigg que escribió Illusion and reality 74; Julian Bell conocido como el poeta de Bloomsbury; Wyston Hugh Auden poeta que publica un largo poema titulado Spain75, editado en París en la publicación Los poetas del mundo defienden al pueblo español, de Nancy Cunnard y Pablo Neruda; el sobrino de Churchill, Esmond Romilly que publica la dura batalla de Boadilla ${ }^{76}$; Humphrey Slater publica la novela The heretics 77 donde critica el papel que jugaron los comunistas en la guerra; Thomas Henry Wintringham Corresponsal del Daily worker y autor d'English $\operatorname{captain}^{78}$; Laurie Lee con su obra $A$ moment of war79, donde se desprenden algunas dudas cronológicas sobre su participación en las Brigadas Internacionales.

72 SOMMERFIELD, John, Volunteer in Spain, New York, A. A. Knopf, 1937.

73 SLOAN, Pat (ed.), John Cornford: a memoir, (Re-issued) London, Cape, 1938.

74 CAUDWELL, Christopher, Illusion and reality: a study of the sources of poetry, London, Lawrenca \& Wishart, 1946.

75 AUDEN, Wyston Hugh, Spain, London, Faber \& Faber, 1937.

76 ROMILLY, Esmond, Boadilla: a personal record of the English Group of the Thaelmann Battalion of the International Brigade in Spain, cit..

77 SLATER, Humphrey, The heretics : a novel, London, Secker \& Warburg, 1946.

78 WINTRINGHAM, Tom, English captain... London, Faber and Faber, 1939.

79 LEE, Laurie, A moment of war: a memoir of the Spanish Civil war, New York, The New Press, 1994. 
Erróneamente, muchos escritores ingleses han sido vinculados a las Brigadas Internacionales cuando en realidad sólo vinieron a España como observadores, muchos de ellos enviados por el Partido Comunistas inglés o bien como autores "turistas". Como son: Stephen Spender autor de Poems for Spain ${ }^{80}$. Louis MacNeice con sus memorias $^{81}$. Arthur Koestler que escribe sus experiencias en Spanish testament ${ }^{82}$. Frank Pitcairn (Claud Cockburn) con sus memorias ${ }^{83}$. John Langdon-Davis y Frank Jenikell, autores de Behind the Spanish barricades ${ }^{84}$ y The civil war in Spain ${ }^{85}$. Para terminar este apartado de escritores ingleses, debemos citar a George Orwell que, aunque nunca se alistó en las Brigadas Internacionales, no podemos pasar por alto que es el autor de las dos obras más emblemáticas que la literatura extranjera ha producido sobre la Guerra Civil en Cataluña ${ }^{86}$ y sobre les Brigadas Internacionales ${ }^{87}$.

\subsection{Irlandeses}

La Guerra Civil española despertó poco interés entre los escritores irlandeses y es por este motivo que las pocas fuentes que existen sobre el conflicto español son de una calidad bastante dudosa. No obstante, unos mil irlandeses participaron en la guerra, setecientos en el bando nacional y unos doscientos en el bando republicano. El general Eoin O’Duffy, escribe Crusade in Spain ${ }^{88}$ donde apunta el escaso acierto que tuvieron los brigadistas irlandeses en la contienda española. A parte de 'O'Duffy, dos poetas irlandeses que participaron en la guerra a favor de la causa republicana fueron Charles Donelly con su antología de Poems for Spain y en The heart of Spain ${ }^{89}$, y Charles Ewart Milne con sus relatos publicados en el Ireland today ${ }^{\circ}$ y los poemas de Letter from Ireland ${ }^{91}$. En cuanto a Paul Vincent Carroll, destaca su obra de teatro The wise

8o SPENDER Stephen, etc. (eds.), Poems for Spain, London, Hogarth Press, 1939.

81 MACNEICE, Louis, The strings are false: an unfinished autobiography, New York, Oxford University Press, 1966.

82 KOESTLER, Arthur, Spanish testament, London, Left Book Club Edition - Gollancz, 1937.

83 PITCAIRN, Frank, In time of trouble : an autobiography, London, R. Hart-Davis, 1956.

84 LANGDON-DAVIES, John, Behind the Spanish barricades, London, Secker \& Warburg, 1936.

85 JENIKELL, Frank, The civil war in Spain, London, V. Gollancz - ltd., 1938.

86 ORWELL, George, Homage to Catalonia, London, Secker \& Warburg, 1951.

87 HEMINGWAY, Ernest, For whom the bell tolls, New York, Charles Scribner's Sons, 1940.

88 O’DUFFY, Eoin, Crusade in Spain. London, Brown and Lolan, 1938.

89 LEHMANN John (eds.), Poems for Spain. Stephen Spender, London, The Hogarth Pres, 1939; BESSIE, Alvah Cecil, The heart of Spain: anthology of fiction, non-fiction and poetry, New York, Veterans of the Abraham Lincoln Brigade, 1952.

90 «Medley in Spain», Ireland today, sept. 1937, pp.41-48; «Escape», Ireland today, des. 1937, pp. 49-54; «The statue», Ireland today, mar. 1938, pp. 231-238.

91 MILNE, Charles Ewart, Letter from Ireland, Dublin, The Gayfield Press, 1940. 
have not spoken ${ }^{92}$ donde habla de un excombatiente de la guerra de España, Francis MacElroy, que el año 1941 se dedica a traficar con armas bajo las órdenes del IRA.

\subsection{Italianos}

La producción literaria italiana sobre el conflicto bélico español es muy escasa, especialmente por lo que respecta a los voluntarios a favor de la causa republicana, durante los años de la guerra y también posteriormente. En las filas de los italianos antifascistas se encuentran pocos escritores reconocidos, el más importante de ellos por el tema que nos ocupa es Luigi Longo autor de Le Brigate Internazionali in Spagna ${ }^{93}$. $\mathrm{Su}$ obra es uno de los mejores libros de memorias brigadistas que se ha escrito, aunque no debemos perder de vista el hecho que Longo era un Inspector General de las Brigadas y en consecuencia la carga subjetiva en sus páginas es muy importante. Otros autores son Camillo Berneri; Randolfo Pacciardi que escribe Il Battaglione Garibaldi 94 donde explica las aventuras de éste batallón italiano en España. Pietro Nenni que fue el Comandante de la Compañía del Batallón Garibaldi y escribió Spagna95, obra muy crítica con los anarquistas que los responsabiliza de los sucesos de mayo de 1937 y cree imprescindible la colaboración con la burguesía republicana. y Carlo Rosselli. Todos ellos famosos por el papel jugado durante la guerra y la posguerra, militantes antifascistas convencidos y verdaderos interlocutores en transmitir sus impresiones de lo acontecido. Después de la guerra, podemos destacar dos autores de izquierdas que han escrito sobre la Guerra Civil, especialmente en sus cuentos: Francesco Jovine y Leonardo Sciascia.

\subsection{Franceses}

Entre los autores franceses destacan Simone Weil con su diario ${ }^{96}$ el libro que mejor nos acerca a su paso por España y su alistamiento en una unidad internacional de la columna Durruti en agosto de 1936. Benjamin Péret que publicó Je ne mange pas de ce

92 CARROLL, Paul Vincent, The wise have not spoken: a drama in three acts, London, MacMillan, 1947.

93 LONGO, Luigi, Le Brigate Internazionali in Spagna, Roma, Editori Riuniti, 1956.

94 PACCIARDI, Randolfo, Il Battaglione Garibaldi: volontari italiani nella Spagna repubblicana, Roma, La Lanterna, [1938?].

95 NENNI, Pietro, Spagna, Milano, Avanti!, 1958.

96 WEIL, Simone, Cahiers, 3 vols, Paris, Plon, 1951-1956. 
pain-là 97 y Je sublime ${ }^{98}$. Louis Aragon, uno de los principales organizadores del Congreso de Escritores Antifascistas. Todos estos autores se incorporaron a las filas de las Brigadas Internacionales y, si bien sí escribieron sobre la guerra, sus producciones literarias no contienen ninguna obra relacionada directamente con el cuerpo de voluntarios.

\subsection{Norteamericanos}

A principios de los años 30, muchos escritores e intelectuales americanos, decepcionados por la situación económica y social de su país, aceptaron con entusiasmo el radicalismo comunista. El marxismo se convirtió para ellos en una ideología que explicaba el declive de las instituciones capitalistas y ofrecía nuevas esperanzas para que, en un futuro, la fraternidad universal y la justicia social substituyeran al capitalismo burgués americano. Destacan los artículos que publicaron periodistas como Herbert Matthews, Vincent Sheean, Lawrence Fernsworth y Jay Allen, apoyando a la causa republicana explicando los bombardeos de Gernika, Barcelona, Almería y Badajoz y las atrocidades de la aviación alemana e italiana; la oposición de un importante número de intelectuales a la política de neutralidad marcada por el gobierno americano; la proyección de películas pro republicanas como Blockade y The Spanish earth consiguiendo un lleno total; y las conferencias de André Malraux y del novelista inglés Raph Bate por todo el país. Entre los escritores americanos y brigadistas destacan: Alvah Bessie con su crónica Men in battle (1939), traducida al español como Hombres en guerra ${ }^{99}$, su actividad posterior a través de los Veteranos de la Brigada Abraham Lincoln y su enfrentamiento con el Comité del Congreso sobre Actividades Antiamericanas en su novela de 1957 The Un-Americans ${ }^{10 o}$, han sido hechos decisivos para convertirlo en abanderado del legado brigadista americano. Robert Colodny autor de The struggle for Madrid ${ }^{101}$ que es la historia militar de la batalla más importante de la Guerra Civil, perfectamente reconstruida y documentada por un autor que la vivió. Frank Glasgow Tinker piloto de la patrulla norteamericana de "Chatos" de la escuadrilla de "Lacalle" y de "Moscas" y en su libro

\footnotetext{
97 PÉRET, Benjamin, Je ne mange pas de ce pain-là, Paris, Éditions Surréalistes, 1936.

98 PÉRET, Benjamin, Je sublime, 1 vol, Paris, Éditions Surrealistes, 1936.

99 BESSIE, Alvah Cecil, Hombres en guerra: historia de norteamericanos en España, México, Era, 1969.

100 BESSIE, Alvah Cecil, The Unamerican, New York, Cameron Associates, 1957.

101 COLODNY, Robert Garland, The struggle for Madrid: the central epic of the Spanish conflict: 1936-37, New York, Paine-Whitman, 1958.
} 
Some still live ${ }^{102}$ explica las dificultades de la lucha aérea siempre en inferioridad numérica y cualitativa.

\subsection{Alemanes}

La guerra de España se convirtió en un reclamo para emigrantes alemanes de extrema izquierda, comunistas y anarquistas que llegaron para alistarse en las Brigadas Internacionales. Entre ellos encontramos un número importante de escritores, de los cuales los más representativos son: Gustav Regler con su novela autobiográfica Das grosse Beispiel (La gran cruzada) ${ }^{103}$. Ludwig Renn escribió en $1955^{104}$ una crónica de guerra, propia de un corresponsal.

\subsection{Soviéticos}

Entre los escritores soviéticos destacan: Mikhail Koltsov que es el ejemplo más representativo de los escritores soviéticos de las Brigadas Internacionales. Considerado "el hombre de Stalin", fue corresponsal del periódico Pravda y consejero político del gobierno republicano en España y su obra más importante es su Diario ${ }^{105}$. Ilya Ehrenburg viene a España en 1936 como corresponsal del periódico Izvestia y destacan sus memorias son una fuente representativa de su vida como corresponsal ${ }^{106}$.

Entre otros escritores están el argentino Raúl González Tuñón; los cubanos Pablo de la Torriente Brau con sus crónicas ${ }^{107}$; Juan Marinello que fue el ideólogo hispanoamericano más comprometido en la defensa de España ${ }^{108}$; y el checo Arthur G. London que publica el libro La confesión : l'aveu ${ }^{109}$.

102 TINKER, F. G., Some still live: experiences of a fighting-plane pilot in the Spanish war, London, Lovat Dickson, 1938.

103 REGLER, Gustav, The great crusade, New York, Toronto, Longmans Green and Co., 1940.

104 RENN, Ludwig, Der Spanische Krieg, Berlin, Aufbau-Verlag, 1956, p. 383.

105 KOLTSOV, Mijail, Diario de la guerra española, Madrid, Akal, 1978.

106 EHRENBURG, Ilya, Corresponsal en España. Barcelona, Prensa Ibérica, 1998.

107 TORRIENTE BRAU, Pablo, En España, peleando con los milicianos, México, Grijalbo, 1972.

108 MARINELLO, Juan, Dos discursos de Juan Marinello al servicio de la causa popular, París, Comité Ibero-Americano, 1937.

109 LONDON, Arthur, La confesión, l'aveu: en el engranaje del proceso de Praga, Madrid, Ayuso, 1970. 


\section{Las fuentes: análisis y tipología}

Después de analizar toda la producción sobre las Brigadas Internacionales y los Brigadistas, y dado que este estudio forma parte de la creación de un sistema de información digital, presentamos los resultados del vaciado sistemático de una muestra significativa de dichas fuentes sobre las Brigadas Internacionales. Partiendo de esta metodología, hemos analizado las fuentes y hemos procedido a establecer su tipología. La tipología de la producción bibliográfica sobre el tema de las Brigadas Internacionales nos aporta la información que necesitamos para configurar el sistema de información. Y hemos establecido cinco grandes grupos de fuentes:

1. Monografías con las obras generales, los estudios parciales sobre algún aspecto de las Brigadas Internacionales, las memorias y los relatos personales.

2. Publicaciones periódicas con la prensa diaria, la interbrigadista y la política.

3. Publicaciones gráficas y audiovisuales con los carteles, fotografías, películas y todo el material gráfico sobre el tema.

4. Manuscritos con la documentación personal de los brigadistas, sus familias y los archivos de las Brigadas y de las asociaciones de familiares y simpatizantes de las Brigadas Internacionales.

5. Recursos web: archivos y bibliotecas, páginas web de los brigadistas, páginas web de asociaciones, páginas web de las Brigadas, bases de datos sobre historia contemporánea, y portales web dedicados a la Guerra Civil española.

Para tipificar estas fuentes hemos seleccionado una muestra de fuentes primarias que sea suficientemente significativa para mostrar un corolario completo de datos, tendencias, ideologías, procedencias geográficas y función de cada fuente. Entre las obras generales sobre las Brigadas Internacionales la obra que constituye el punto de partida es la de Andreu Castells ya mencionada, puesto que cumple con todos los requisitos necesarios y especialmente porque cuenta con un índice onomástico muy elaborado y muy completo. En cuanto a las obras generales sobre la Guerra Civil, únicamente hemos incorporado aquellas que incluyen un capítulo importante sobre las Brigadas Internacionales y aparecen referenciadas como partes de una monografía o como artículo de revista para remarcar la importancia de aquel capítulo dentro de la 
obra completa como son: La Intervención extranjera en la guerra de España ${ }^{110}$, Les Brigades Internationales ${ }^{111}$, La guerra : las Brigadas Internacionales ${ }^{112}$, la obra de Martínez Bande ${ }^{113}$, Historia política y militar de las Brigadas Internacionales ${ }^{114}$ y las aportaciones recientes de Rémi Skoutelsky ${ }^{115}$.

En lo referente a los estudios parciales que son todas aquellas fuentes que tratan el tema brigadista desde diversas ópticas hemos seleccionado como referente las de la Brigada Abraham Lincoln, sobre la cual se han escrito, entre muchas otras, títulos como: The odyssey of the Abraham Lincoln Brigades: americans of the Abraham Lincoln Brigade ${ }^{116}$, The Lincoln battalion: the story of the Americans who fought in Spain in the International Brigades ${ }^{117}$ y la obra de Arthur H. Landis ${ }^{118}$. También hemos incorporado las memorias a pesar de su potente componente de subjetividad porque son una fuente imprescindible para conocer lo que sucedió de primera mano, más o menos desvirtuado. Como señala Pierre Vilar119: "son esenciales, ya sean de dirigentes o de gente de base, para establecer la sicología del que lo ha vivido”. Asimismo, hemos seleccionado entre el volumen de memorias sobre temática brigadista los autores que más han publicado como son los poloneses ${ }^{120}$, $\operatorname{checos}^{121}$, yugoslavos ${ }^{122}$, y soviéticos, y también americanos ${ }^{123}$ e ingleses ${ }^{124}$, que han dejado la huella literaria de su paso por las Brigadas Internacionales en forma de relato personal.

110 SALAS LARRAZABAL, Jesús María, Intervención extranjera en la guerra de España, Madrid, Editora Nacional, 1974.

111 DELPERRIE DE BAYAC, Jacques, Les Brigades Internationales, París, Fayard, 1968.

112 FUSTER, Francisco, La guerra: las Brigadas Internacionales, Albacete, Artes Gráficas Quintanilla, 1985.

113 MARTÍNEZ BANDE, José Manuel, Brigadas Internacionales, Barcelona, Caralt, 1972.

114 ÁLVAREZ, Santiago, Historia política y militar de las Brigadas Internacionales, Madrid, Compañía Literaria, 1996.

115 SKOUTELSKY, Rémi, Novedad en el frente: las Brigadas Internacionales en la Guerra civil, Madrid, Temas de Hoy, 2006.

116 CARROLL, Peter N., The odyssey of the Abraham Lincoln Brigades: Americans of the Abraham Lincoln Brigade, Stanford (Calif.), Standford University Press, 1994.

117 ROLFE, Edwin, The Lincoln Battalion: the story of the Americans who fought in Spain in the International Brigades, New York, Veterans of the Abraham Lincoln Brigade, 1939.

118 LANDIS, Arthur H., The Abraham Lincoln Brigade. New York, The Citadel Press, 1967.

119 VILAR, Pierre, «Historia e historiografía de la Guerra Civil española: algunas reflexiones metodológicas», (dins. Pierre Broué, Roland Fraser, Pierre Vilar), en Metodología histórica de la guerra y revolución españolas, Barcelona, Fontamara, 1980.

120 AJZNER, Seweryn, Polska a wojna domowa $w$ hispanii: 1936-1939, Warszawa, Panstwowe Wydawnictwo Naukowe, 1968.

121 NETOPIL, Ludvík, Ze spanelskúch zákopu, Holesov, Vytiskla Grafia Brno, 1969.

122 ERGAN, Joze, Od marezig do Madrida, V. Ljubljani, Borec, 1962.

123 COLODNY, Robert Garland, Spain: the glory and the tragedy, New York, Humanities, 1970.

124 GURNEY, Jason, Crusade in Spain, London, Faber and Faber, 1974. 
La prensa es otra de las fuentes fundamentales pero muy problemática desde el punto de vista historiográfico, porque tal como señala Pierre Vilar ${ }^{125}$ : «no existe nada más difícil que la utilización de la prensa para el historiador». Después de analizarla, la prensa no es una fuente útil para extraer una conclusión objetiva de los hechos que explica, especialmente en época de conflicto armado. No podemos olvidar las opiniones, las proclamas a uno u otro bando y la ideología o el partido político del cual es portavoz. Lo que hemos realizado en este estudio es la selección de diversos diarios y publicaciones periódicas y hemos vaciado sistemáticamente todos sus números entre los años del conflicto bélico para extraer toda la información existente sobre brigadistas y las Brigadas. Por otra parte, disponemos de la prensa interbrigadista. Se trata de una fuente puramente militar, elaborada en el mismo campo de batalla, que explica el día a día en el frente, con todo los problemas que conlleva la formación de un ejército no profesional, que proviene de diversos países, con ideologías e intereses distintos y una único punto en común, su adhesión al conflicto al lado del ejército regular de la II República. Estas publicaciones interbrigadistas se caracterizan por su idea de ser un referente y una consigna clara para los combatientes, en este caso los voluntarios internacionales. Otro aspecto importante que las define es la diversidad idiomática con la cual se editaron. El ejemplo más importante es Le volontaire de la libertér ${ }^{126}$, que se editó en las cinco lenguas más importantes de los combatientes extranjeros: francés, inglés, alemán, italiano y polaco. Se consideró la publicación "madre" de todas las Brigadas Internacionales, a partir de la cual se fueron editando el resto de publicaciones siguiendo la propia estructura miliar brigadista de divisiones, regimientos, brigadas, batallones, compañías y artillería.

Sin embargo la prensa interbrigadista presenta un doble problema. Por un lado, no disponemos de colecciones completas de todas estas colecciones. Lo que ha quedado está disperso por archivos y hemerotecas de todo el mundo y se trata de colecciones incompletas. Por otro lado, y suponiendo que dispusiéramos de las colecciones íntegras, no serían, aunque en un principio lo podríamos pensar, una fuente de información clave para proceder al vaciado documental y obtener una gran número de información sobre las biografías de los brigadistas. Después del análisis de algunos números de algunos títulos, nos reafirmamos en esta hipótesis: no aparecen ni listados de nombres, ni encuadramientos militares nominativos, ni datos biográficos relevantes.

\footnotetext{
125 Pierre VILAR. «Historia e historiografía de la Guerra Civil española: algunas reflexiones metodológicas», cit., p. 42.

${ }_{126}$ Le volontaire de la liberté: órgane des Brigades Internationales, [Ed. française], 1937, 4 mars-1938, 1 juin.
} 
Las fuentes gráficas y audiovisuales son la tipología más compleja de análisis porque la narrativa de su discurso no es siempre explícita. Hemos estudiado carteles, fotografías y películas. Después de un primer análisis de estas fuentes hemos decidido su digitalización y análisis por expertos que forman parte del grupo de investigación que desarrolla este proyecto: historiadores, lingüistas y expertos en cinematografía y museografía. Para desarrollar la selección de estas fuentes hemos vaciado la colección de carteles y fotografías del Pavelló de la República (Universitat de Barcelona) y hemos estudiado los nodos y otras películas disponibles.

Los manuscritos y los archivos personales de los brigadistas han sido estudiados a través de las fuentes de la Asociación Amigos de las Brigadas Internacionales. Esta documentación es la más sensible y compleja por ser una documentación personal (cartas, comunicados,...), que está sometida a la legislación sobre la privacidad y la difusión, y que es muy heterogénea e irregular.

$\mathrm{Y}$ finalmente los recursos web es un tipo de fuente que requiere un vaciado exhaustivo y el contraste con otras fuentes ya mencionadas para no incurrir en la repetición. Entre todos los recursos cabe destacar que los más relevantes son las pocas páginas web de los brigadistas, las páginas web de algunas de las Brigadas, y las páginas web de las asociaciones.

Y finalmente para refinar la selección de las fuentes hemos realizado búsquedas en las principales bases de datos ${ }^{127}$ del ámbito de las humanidades que nos han ayudado a contrastar y confirmar nuestra propuesta de tipología de las fuentes sobre las Brigadas Internacionales.

Después de haber concluido con la tipología de las fuentes hemos creado un prototipo de base de datos "Fuentes" que incluye una muestra de 514 obras, y que nos permite extraer los primeros datos cuantitativos y cualitativos sobre la producción especializada en las brigadas Internacionales. Las primeras conclusiones son:

1. Hemos localizado fuentes publicadas en 35 países diferentes, destacando en número las publicadas en España. En cuanto a las obras editadas en el exterior, la mayor producción bibliográfica tiene lugar en Estados Unidos, Gran Bretaña e Italia. Destacan las pocas fuentes existentes de los países de la órbita soviética, y también es importante la existencia de libros editados en países como Dinamarca, Israel, México o Suiza. Sin embargo, hay un porcentaje bastante elevado de fuentes sin lugar de edición.

127 Hemos vaciado exhaustivamente las siguientes bases de datos: ARCE, ISOC (CSIC), Isi Web of Knowledge, Historical Abstracts, Wilson Web y Fons d'Història Local. 
2. Se han localizado fuentes escritas en 26 idiomas diferentes y 3 obras multilingües. Destacan sobre todo las españolas, seguidas de las inglesas (que engloban tanto las obras editadas en Gran Bretaña como en Estados Unidos y Canadá), las italianas y las francesas. Es de destacar el gran número de obras escritas en alemán y no publicadas en Alemania.

3. Los años de mayor producción bibliográfica son los de la guerra (1936-1939). Hecho lógico si pensamos en la gran cantidad de memorias y relatos personales escritos por los propios protagonistas de la contienda. Sin embargo, no será hasta el final del franquismo (1975) que las Brigadas Internacionales volverán a ser objeto de estudio debido a la muerte del dictador, sobretodo con la desaparición de la censura y el uso de técnicas historiográficas más objetivas.

4. La mayor parte de la producción bibliográfica sobre Brigadas Internacionales la constituyen las novelas, las memorias, y casi con el mismo porcentaje las obras que tratan de los brigadistas de un país concreto. Es interesante destacar, no por el número sino por la originalidad, las obras que hablan de aspectos muy determinados del fenómeno brigadista como son por ejemplo la sanidad, las mujeres, los niños, y multiplicidad de otros temas.

Nuestro proyecto para la creación de un portal semántico sobre las Brigadas Internacionales (SIDBRINT) incorpora todas las fuentes mencionadas y su selección sigue la metodología explicada en este artículo. El portal web estará disponible, en fase de pruebas, el mes de septiembre del 2011 como homenaje al 75 aniversario de las Brigadas Internacionales ${ }^{128}$.

128 URL: < http://www.sidbrint.org > 


\section{Conclusión}

La invisibilidad de las fuentes en los sistemas digitales que existen hoy en la Web, la falta de accesibilidad al conocimiento que esas fuentes ofrecen sobre un fenómeno histórico único en la historia europea, y la necesidad de recuperar la identidad colectiva sobre la memoria histórica de la Guerra Civil española ha llevado a las autoras de este articulo a la creación de prototipos para el diseño de un sistema digital que recoge y ofrece todas las fuentes documentales sobre las Brigadas Internacionales y los Brigadistas. El sistema de información digital, en formato portal web, se propone participar en la construcción de la historia sobre los brigadistas y ayudar en la desaparición del silencio, los mitos y las leyendas que con su no a la historia crearon algunas de las ideologías imperantes en España y Europa durante buena parte del siglo XX. 


\section{* Autores}

*Lourdes Prades-Artigas es Doctora en Historia Contemporánea y Directora de la Biblioteca del Pavelló de la República (Universitat de Barcelona). Actualmente participa en la investigación en el Proyecto: "La memoria histórica y las Brigadas Internacionales: diseño de un sistema digital para la transferencia del conocimiento sobre el patrimonio histórico español" (Reconocido como Proyecto I+D+I del Ministerio de Ciencia y Tecnología, (HAR2010-20983subprograma HIST). Entre sus principales publicaciones recordamos: «I Miliziani delle Brigate Internazionali della Guerra civile spagnola e la memoria storica: SIDBRI, un sistema di informazione digitale», Spagna Contemporanea, núm. 36, 2009, pp. 193-216 (con Montserrat Sebastiá); «El fons documental de l'arxiu-biblioteca del Pavelló de la República de la Universitat de Barcelona», en Espai, art i memòria: el Pavelló de la República (París 1937-Barcelona 2007), Barcelona, Publicacions i Edicions de la Universitat de Barcelona, 2008, pp. 103-115 (con Olga Giralt); Els Brigadistes de la Guerra Civil espanyola en les fonts documentals: un prototipus de sistema d'informació digital, Tesi doctoral. Barcelona, Universitat de Barcelona, 2008; «Fons hemerogràfic de la Biblioteca del Pavelló de la República (Universitat de Barcelona): VI Colloqui Aula d'Història del Periodisme Diari de Barcelona», Treballs de comunicació: Societat Catalana de Comunicació (Barcelona), núm, 18, (desembre) 2003, pp. 193-196. En publicación: “Forjant’ El imperio del libro católico y españolísimo" l'edició a Catalunya durant els primers anys del Franquisme» dins Congrés Internacional La dictadura franquista: la institucionalització d'un règim, Barcelona, Universitat de Barcelona, Facultat de Geografia i Història, 2011.

URL: < http://studistorici.com/progett/autori/\#GonzálezMartín >

**Montserrat Sebastiá-Salat, es Profesora Titular de la Universidad de Barcelona (Departamento de Biblioteconomía y Documentación), y es Investigadora Principal del Proyecto: "La memoria histórica y las Brigadas Internacionales: diseño de un sistema digital para la transferencia del conocimiento sobre el patrimonio histórico español". Entre otros ha publicado: «Asociaciones profesionales y la creación de las Escuelas de Biblioteconomía y Documentación», Los estudios de Biblioteconomía y documentación, Madrid, 1983 (Fund. Univ. Empresa, 52); M. Thesaurus d'Història Social de la Dona, Barcelona, 1988; «La dona i la institucionalització de l'educació», Més Enllà del Silenci, Les Dones a la Història de Catalunya, Barcelona, 1988, p. 206-226 (con Cortada Ester); «Los Modelos Curriculares de las Tecnologías de la Información en el área de las Ciencias de la Documentación», en Métodos Didácticos en Biblioteconomía y Documentación, Universidad Complutense, Madrid, 1997, p. 181-197; «L'Arquitectura de la Informació i el Disseny Curricular: Competitivitat i Discontinuïtat», Bibliodoc Anuari/Anuario del Col.legi Oficial de Bibliotecaris de Catalunya, Barcelona, 2003, p. 51-75; «La Transformación de los Archivos y de la Archivística: el nuevo paradigma desde la 
$\overline{\text { hibridez a la metadisciplina y la metacomunicad profesional», Tabula, Revista de Archivos de }}$ Castilla y León, Asociación de Archiveros de Castilla y León, vol. 5, núm. 12, 2009, pp. 17-30.

URL: < http://studistorici.com/progett/autori/\#GonzálezMartín >

\section{Per citare questo articolo:}

PRADES-ARTIGAS, Lourdes, SEBASTIÁ-SALAT, Montserrat, «Fenomenología y Guerra Civil española: La visibilidad de las fuentes documentales sobre las Brigadas Internacionales (1937-2011)», Diacronie. Studi di Storia Contemporanea: Spagna Anno Zero: la guerra come soluzione, 29/07/2011,

URL:<http://www.studistorici.com/2011/07/29/prades_numero_7/ >

Diacronie Studi di Storia Contemporanea $\beta$ www.diacronie.it

Risorsa digitale indipendente a carattere storiografico. Uscita trimestrale. redazione.diacronie@hotmail.it

Comitato di redazione: Marco Abram - Giampaolo Amodei - Jacopo Bassi - Luca Bufarale - Alessandro Cattunar - Alice De Rensis Barbara Galimberti - Deborah Paci - Fausto Pietrancosta - Martina Sanna - Matteo Tomasoni - Luca Zuccolo

Diritti: gli articoli di Diacronie. Studi di Storia Contemporanea sono pubblicati sotto licenza Creative Commons 2.5 Possono essere riprodotti a patto di non modificarne i contenuti e di non usarli per fini commerciali. La citazione di estratti è comunque sempre autorizzata, nei limiti previsti dalla legge. 